\title{
INTER-DOMAIN ROUTING IN OPTICAL NETWORKS
}

\author{
Américo Muchanga ${ }^{1}$, Lena Wosinska ${ }^{2}$, Fredrik Orava ${ }^{3}$, Joanna Haralson ${ }^{4}$ \\ Royal Institute of Technology, KTH/IMIT, Sweden, \\ lamerico@imit.kth.se, ${ }^{2}$ Lena.Wosinska@imit.kth.se, ${ }^{3}$ fredrik@it.kth.se, ${ }^{4} i t 03$ jdr@it.kth.se
}

\begin{abstract}
In this paper we present a mechanism for obtaining an abstraction of network topology in optical networks in order to compute an end-to-end lightpath across multiple domains.
\end{abstract}

\section{INTRODUCTION}

The main benefit of the Multiprotocol Label Switching (MPLS) is to provide traffic engineering in IP networks. The connectionless operation of IP networks becomes more like a connection-oriented network where the path between the source and the destination is pre-calculated based on user specification.

Generalized Multiprotocol Label Switching (GMPLS) extends MPLS to provide the control plane (signaling and routing) for devices that switch in any domain, i.e. packet, time, wavelength and fiber. This common control plane is expected to simplify network operation and management by automating end to end provisioning of connections, managing network resources, and providing the level of QoS that is expected in the new, sophisticated applications.

Thus future data and telecommunication networks are likely to consist of elements that will use GMPLS to dynamically provision resources and to provide network survivability using protection and restoration techniques.

There is also a great interest in extending IP-based protocols to control optical networks. To replace the existing solutions the new optical transport networks must provide similar or higher QoS, protection and restoration mechanisms that are available in current techniques. This implies that network operators have to be able to provide end-to-end QoS guarantees for its customers. Thus the network operators or carriers need to have traffic engineering capabilities to set lightpaths in 
networks that are beyond their administration domains, which means that they have to be able to exchange resources and topology information through an Exterior Gateway Protocol (EGP).

Domain boundaries exist for the purpose of abstraction from irrelevant details, e.g. domain related information, to the outside world. This information might reveal to the network architecture between two competing entities. However it might be vital to enable network or service providers to achieve diversity and protection that is essential for optical networks survivability

There are only a few attempts that address the issues of inter-domain routing and optical networks [1,2]. They are still in the early phases. In order to utilize the full potential of optical networks information about optical network resources has to be conveyed through inter-domain routing protocols. However, the IETF GMPLS architecture draft [3] does not cover extensions for inter-domain routing (e.g. BGP).

This paper addresses the information that has to be shared between domains through an inter-domain signaling mechanism and its level of relevance to support the development of optical networks. This information could be conveyed through traffic engineering extensions to the existing inter-domain routing protocols. Therefore we are proposing a method of network abstraction that would enable extensions to BGP to achieve traffic engineering information exchange required to facilitate the operation of networks. The ability to diversely route optical connections is very important for the reliability and resilience of the optical network.

Our approach is to use BGP for sharing not only the information about network reachability but also to share information about essential data in internal networks that is required to enable peers to setup end-to-end lightpaths with requested quality.

\section{RATIONALE FOR INTER DOMAIN ROUTING IN OPTICAL NETWORKS}

In this paper we concentrate on the signaling and routing issues when networks belonging to different carriers are interconnected. We consider services that make two competing companies to be involved in optical inter-domain routing relationship.

In inter-domain routing relationship today, service providers share basically only the network reachability information. Occasionally they do exchange information that allows them to reach some degree of diversity, in situations where multi-exit discrimination is possible. In these situations the inter-domain routing protocol is not used to carry information that has any significance for lower layers. 
However, inter-domain relationship in optical networks can involve exchange of information that can reveal the network architecture of the competing peers, and

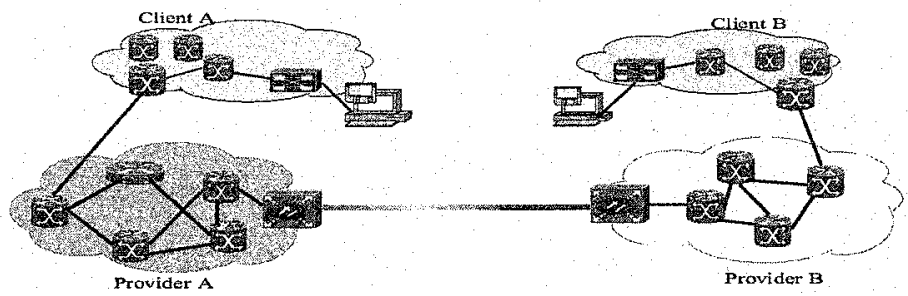

Figure 1. Network model and components of provider networks interconnected through a wavelength routing network

thus affecting the competitive advantage of the participating entities. If end-to-end light path provisioning is to be achieved, service providers need to exchange some information in the interconnecting network interfaces, which is beyond the network reachability information that is carried today.

Figure 1 shows network model and components. We focus on the relationship between provider $\mathrm{A}$ and provider $\mathrm{B}$, and in particular on the cooperation between the edge routers that interconnect the networks of the providers. Figurel shows a situation in which the networks are connected by a point-to-point optical link. Our proposed method, however, is not limited to this simple topology and we consider also more general network topologies.

Note, that if two IP clients are connected through two optical domains (as shown in Figure 1) the conventional IP adjacency for IP layer operations as well as adjacencies for circuit switched operations have to be maintained.

The optical control plane has been proposed in the GMPLS architecture [3]. Protocols and functions of an optical control plane are illustrated in Figure 2. The optical network control plane is divided in routing protocols and signaling protocols. Routing protocols are used for topology and resource discovery and

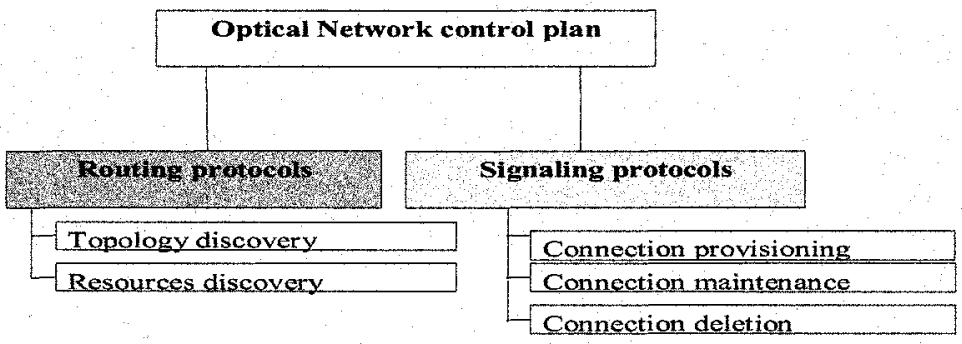

Figure 2. Protocols and functions of an optical control plane 
dissemination while signaling protocols are used for connection provisioning, maintenance and termination.

Topology and resource discovery and dissemination can be done both within domain and between domains. Protocols that achieve that within a domain have been proposed $[4,5]$ and are now going through a maturing phase. They have been presented in a form of extensions to existing protocols such as Open shortest path first (OSPF) and Intermediate System to Intermediate System (IS-IS). These extended protocols have already been implemented in a number of routing engines. However, so far there is no protocol implementation that provides the same functionality for inter-domain operations.

\section{ROLE OF INTER-DOMAIN ROUTING PROTOCOLS}

To support devices that switch in time, wavelength and fiber, MPLS was extended with protocols that advertise the availability of these resources in order to allow the establishment of a label switched end-to-end path. OSPF and IS-IS have been extended to advertise, within a domain, the availability of optical resources in the network (e.g. generalized representation of various link types, bandwidth on wavelengths, link protection type, fiber identifiers). Once that information is known signaling protocols such as RSVP[6] and LDP[7] have been extended for traffic engineering purposes that allow a label-switched path (LSP) to be explicitly specified across the optical core. Figure 3 shows a constraint-based routing flow.

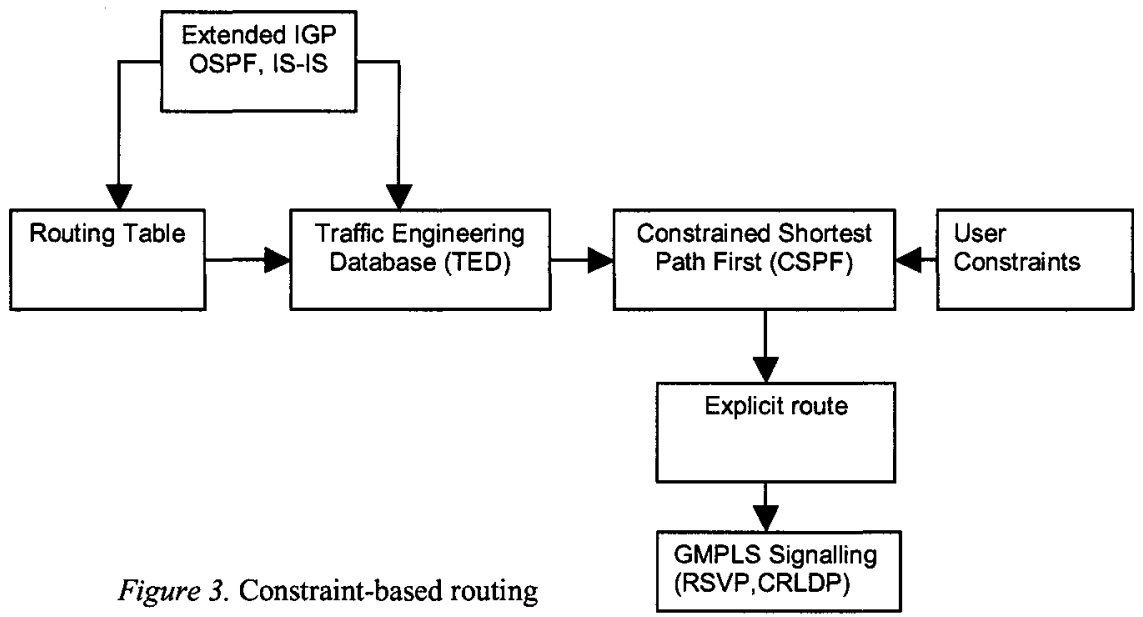

BGP is a path vector protocol, which in the current implementation doesn't include mechanisms to deliver the topology and link status information that is important in computing optical routes. However, BGP could be potentially used to support some or all the services provided by intra-domain routing protocols in order to enable a proper operation of signaling protocols. 


\section{OPERATORS' PERSPECTIVE}

In order to offer similar services as provided today by intra-domain traffic engineering protocols, BGP has to be modified to carry all the database containing link and resources information that is created by these intra-domain routing protocols. In addition it has to support the LSP setup process that is accomplished through GMPLS signaling.

BGP, after receiving information, would have to digest it in the internal network in order to allow internal routers to create a database that reflects the global network encompassing different network operators.

Exporting the link state information from one domain to another domain would consume a lot of resources during the initial flooding process and after a change of the network topology or availability resources. Announcing all this information would make the networks not to scale, because the abstraction used today between domains (i.e., only announce reachable networks and not the full topology) is what makes Internet to be able to scale to its current size. Additionally it would enable the peering entities to gain a detailed view of how the other network is implemented that would require applying expensive routers in order to handle huge amount of information. Getting access to this kind of information would enable one entity to gain competitive advantage by being able to engineer his network to offer superior quality of service guarantees.

The above reasons make it unlikely that network providers will be willing to share the entire view of their network to a competing entity, because it does not scale, it is costly and is not attractive from a business point of view. However, the traffic engineering characteristics of GMPLS and its ability to use one forwarding mechanism for multiple applications makes it an attractive technology that could enable providers that are connected at several points to achieve for instance load balancing in the inter connection links and to design services that are based on differentiated QoS parameters.

Therefore, BGP/GMPLS should be used to allow the peering entities to create an abstracted view of the network and share only a few abstract links or paths in the network that would provide to the peering entities some degree of choice to reach destinations inside or outside that network. Thus, we propose a way to achieve the abstracted topology and how BGP could carry that information.

As mentioned, there are strong reasons why all learned information should not be exported to BGP. Therefore we suggest that a carrier or network provider should create abstract aggregations of virtual domains inside his networks to be advertised to peering Exterior Gateway Protocol (EGP) speakers in order to allow them to have some traffic engineering capabilities. This concept is also raised in [8] and is illustrated in Figure 4 where two providers own a mesh network with links characterized by their resources and capacities.

There are many possible paths to go from say R1 to R6. These possible paths are discovered inside the domain of the providers through the use of any extended Interior Gateway Protocol (IGP). Thus the border routers, e.g. R7 and R6, contain a traffic engineering database describing their view of the network within provider A. Our suggestion is that BGP should, for instance, advertise only two paths to the 
peers. For instance R7 located in provider A could advertise to R8 and R9 located in provider $B$ that $R 1$ can be reached through the path R7-R2-R1. In the same way R6 located in provider A would advertise to R9 located in provider B that R1 can be reached through the path R6-R3-R4-R1. Note that there are more possible paths to go from R6 to R1, for instance, R1 could be reached by the route R6-R5-R4-R1. However, the explicit paths are not exported to the peer, only the knowledge about the existence of two paths, the associated properties and also how to name them should be communicated. In this way some degree of diversity or choice can be offered to the peer provider while maintaining some abstraction within the network domain, enabling the providers to hide the details about their network that they feel would impact their competitive advantage.

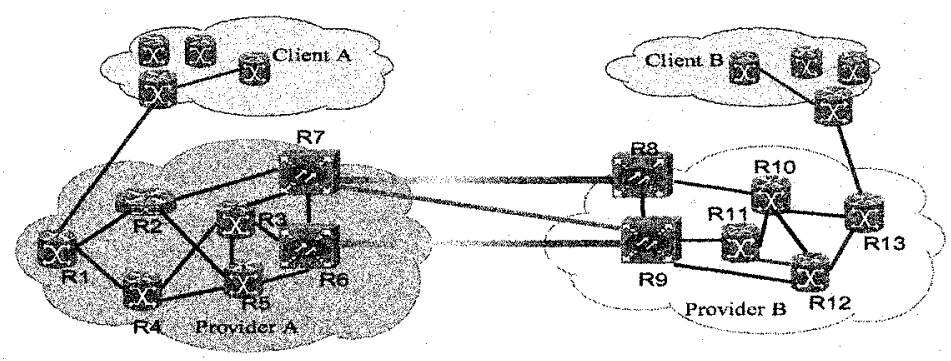

Figure 4. An example of abstraction that border routers could provide to each other

5. MECHANISM FOR CREATION OF ABSTRACTED
PATHS

In order to create the abstracted network topology with only few possible paths between the edge nodes we suggest introducing the following constraints:

1. Node diversity

2. Minimum bandwidth available within the paths

3. Degree of protection

4. Shortest paths

As a result the edge nodes could be asked for instance to compute a shortest path that is node diverse and has a minimum of $10 \mathrm{Mbps}$ with links along the path protected by a dedicated protection.

The idea is that the resulting paths are the ones that could be advertised to other EGB speakers in the border routers. This result could be tightened to a quality of service that might be requested by a peer provider. Assume for instance that client A and B in Figure 4 is one company located in two geographically separated areas. 
In location $\mathrm{A}$ client $\mathrm{A}$ is connected through Provider $\mathrm{A}$ and in location $\mathrm{B}$ client $\mathrm{B}$ is connected through provider B. Providers A and B have to agree on the end-to-end QoS they want to provide to their respective customers. Thus the two providers should decide to advertise to each other paths selected along the constraints that enable them to guarantee the required QoS.

The advantage of this method is that creating the abstracted network topology inside a domain is based on IGP and signaling protocols such as RSVP-TE. As a result the constructed paths are just like any other LSPs and can be treated like any LSPs that have satisfied certain computation criteria.

This procedure can be extended for interconnection of multiple domains. Assume two domains are connected through a third domain as shown in Figure 5. Provider B can advertise to Provider A and C only a limited set of paths to reach both domains. In this scenario we abstract a domain as if it was a node, one can decide to advertise either all possible paths connecting domains $\mathrm{A}$ and $\mathrm{C}$ or only the constrained paths.

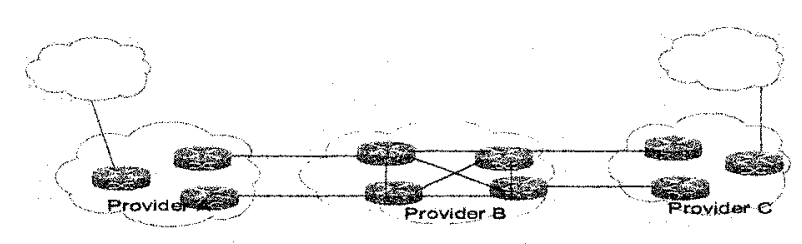

Figure 5. Interconnection between three providers

In this latter case BGP needs to be modified in order to carry only the abstracted information to the peer, which is the main advantage of our proposal.

\section{CONCLUSIONS}

Routing and signaling protocols play an important role in optical networks. We have proposed that Interior gateway protocols can be extended in order to advertise not only link state information that is basically used to provide reachability information but also to convey resource information that is required for traffic engineering. In this way Routing and Signaling protocols can be extended to support packet switching devices as well as time, wavelength and spatial switching devices.

In order to allow for traffic engineering to be done on end-to-end basis across multiple domains link state information as well as traffic engineering (TE) information between domains needs to be exchanged. This should be done through an Exterior Gateway Protocol such as BGP. 
Exporting the entire network map with all TE parameters is not reasonable due to business, operational, capacity and scalability purposes. Therefore we propose a method to obtain an abstraction of the network topology. In this method only a few optional paths satisfying some acceptable QoS guarantees are provided. These QoS can be negotiated between peering entities and used to create the visible LSPs. The paths can be computed using constraints such as diversity, bandwidth and protection.

A similar thinking can be applied for links inside a domain as well as for links connecting domains. In this case a domain is seen as if it was a node with several interfaces that are connected to other domains.

We also propose that once the abstracted set of paths have been obtained and announced to the peers, a streamed down mechanism can be applied in the similar way as presented in [1].

Finally, we believe that the proposed mechanism is promising and we are going to evaluate its performance by OPNET simulations as well as by experiments made on our testbed.

\section{ACKNOWLEDGMENTS}

The authors wish to thank Prof. Björn Pehrson for encouragement, support and for providing the testbed where the optical networking experiments are carried out.

\section{REFERENCES}

[1] Y.Xu, A. Basu, Y.Xue, "A BGP/GMPLS Solution for Inter-domain Optical Networking", I-D, (Expired October 2003)

[2] G. Bernstein, L.Ong, B.Rajagopalan, D.Pendarakis, A.Chiu, F.Hujber, J.Strand, V.Sharma, S.Dharanikota, D.Cheng, R.Izmailov, "Optical Inter domain routing Considerations", I-D, (Expired Augusti 2003)

[3] Eric Mannie (Editor), et.al, "Generalized Multi-protocol Label Switching (GMPLS) Architecture", I-D, Work in Progress (Expired November 2003).

[4] K. Kompella, Y. Rekhter, "OSPF Extensions in Support of Generalized MPLS", I-D, Standards Track, Work in Progress (Expired June 2003).

[5] K. Kompella, Y. Rekhter, "IS-IS Extensions in Support of Generalized MPLS", I-D, Work in Progress (Expired June 2003)

[6] L. Berger, "RFC 3473 - Generalized Multi-Protocol Label Switching (GMPLS) Signaling Resource ReserVation Protocol-Traffic Engineering (RSVP-TE) Extensions", Standards Track, January 2003

[7] P. Ashwood-Smith and L. Berger, "RFC 3472- Generalized Multi-Protocol Label Switching (GMPLS) Signaling Constraint-based Routed Label Distribution Protocol (CR-LDP) Extensions", Standards Track, January 2003

[8] G.M.Bernstein, V.Sharma, L.Ong, "Interdomain Optical Routing", Journal of Optical Networking, Feb 2002/Vol. 1, No.2 